\title{
THE EVALUATION OF AN EFFECTIVE DOSAGE OF CARONAMIDE (4-CARBOXYPHENYLMETHANESULFONANILIDE) FOR THE SUPPRESSION OF TUBULAR EXCRETION OF PENICILLIN IN CHILDREN 1, 2, 3,4
}

\author{
BY F. BRUCE CORNEAL, GAVIN HILDICK-SMITH, MARY B. FELL, \\ AND T. F. MCNAIR SCOTT
}

\begin{abstract}
(From The Children's Hospital of Philadelphia [Department of Pediatrics, School of Medicine, University of Pennsylvania], Philadelphia)
\end{abstract}

(Received for publication February 2, 1948)

It has been shown by Beyer et al. in a recent series of papers ( 1 to 4 ) that the compound 4-carboxyphenylmethanesulfonanilide, known as Caronamide, is effective in increasing the penicillin plasma concentration and in prolonging the period of time over which detectable penicillin plasma concentrations are maintained following administration of penicillin, orally and by venoclysis, to dogs. In these experiments it was demonstrated that the renal tubular excretion of penicillin could be completely suppressed (2) when the antibiotic was given concomitantly with adequate amounts of Caronamide.

In a series of experiments in children (5), Caronamide used in conjunction with penicillin administered orally was effective in increasing the penicillin plasma concentration from 2.8- to 14.5fold over the penicillin controls and was also found to be of a low order of toxicity at the dosages employed.

The purpose of the present research was to determine an optimum dose of Caronamide for children.

\section{METHODS}

Method of administration. Afebrile convalescent patients, aged two to nine years, with normal renal function were selected. Single doses of approximately 300,000 units of penicillin/sq. M. of body surface (equivalent to approximately 10,000 units $/ \mathrm{Kg}$. of body weight) were given intramuscularly as a control, and blood was drawn

1 This study was supported by a grant-in-aid from Sharp \& Dohme, Inc.

2 The Caronamide used in this study was supplied by Dr. Karl Beyer of the Research Department of Sharp \& Dohme, Inc.

8 The Sharp \& Dohme proprietary name for this compound is now "Staticin" Caronamide.

4 Some of the penicillin used in this study was kindly supplied by Commercial Solvents Company, Ltd. for assay at intervals of one-half, 5 one, three, five and seven hours after administration. The penicillin used was the regular crystalline commercial variety containing 90 per cent penicillin G. One of the authors always made the dilutions (with physiologic saline) and administered the dose. At intervals of two days thereafter, the same patients received the same dose of penicillin intramuscularly together with Caronamide orally, the Caronamide dosage being increased on each successive trial. The daily dose of Caronamide ranged from 5 to $20 \mathrm{Gm}$./ sq. M./24 hours. The daily doses of 5,10 , and $15 \mathrm{Gm} . /$ sq. M. were divided into six equal aliquots given at four-hour intervals. Two aliquots were given as priming doses before, and the third simultaneously with the intramuscular dose of penicillin. The largest daily dose, $20 \mathrm{Gm}$./sq. M., was divided into eight equal aliquots given at three-hour intervals with three aliquots given as priming doses, and the fourth with the penicillin. This different interval was used in order to reduce the size of individual aliquots at this high dosage level.

Method of assay. A modified Rammelkamp assay technique (6) was employed, using half-step dilutions of the test plasma with a hemolytic streptococcus ${ }^{6}$ as the test organism.

\section{RESULTS}

The object of this study was to determine how great a dose of Caronamide is required to suppress completely the tubular excretion of penicillin, reducing it to that of glomerular filtration alone. Since the measurement of direct clearance values was impracticable, the results were obtained by indirect means.

Eagle and Newman (7) have shown that the renal clearance of penicillin approximates the total renal plasma flow and is four to five times greater than the glomerular filtration alone. In other words, 20 per cent of the penicillin is excreted by

5 Time at which it was assumed that complete equilibrium between plasma and extracellular fluid had been achieved.

- Kindly supplied by Drs. Verwey and Miller, of Sharp \& Dohme, Inc. 
the glomeruli and 80 per cent by the tubules. As the tubular excretion is decreased the proportion of the penicillin excreted by glomerular filtration is increased, the actual rate of renal clearance of penicillin being proportionately diminished, until 100 per cent of the penicillin is excreted by glomerular filtration, and the rate of plasma clearance becomes constant at approximately one-fifth of the initial rate. In these experiments, the slopes obtained by plotting the levels of penicillin concentration measured were considered as indicating renal clearance of penicillin. ${ }^{7}$ Since the rate of plasma penicillin clearance follows an exponential curve the rates of clearance at different dosages of Caronamide could be compared to the rate of clearance of penicillin alone by comparing the straight line slopes of the decreasing plasma penicillin levels as plotted on semi-logarithmic paper. The minimum dose of Caronamide resulting in a

A.A. 9yrs. Wt.26.36kg. Surfoce Area 1.02 sq.m.

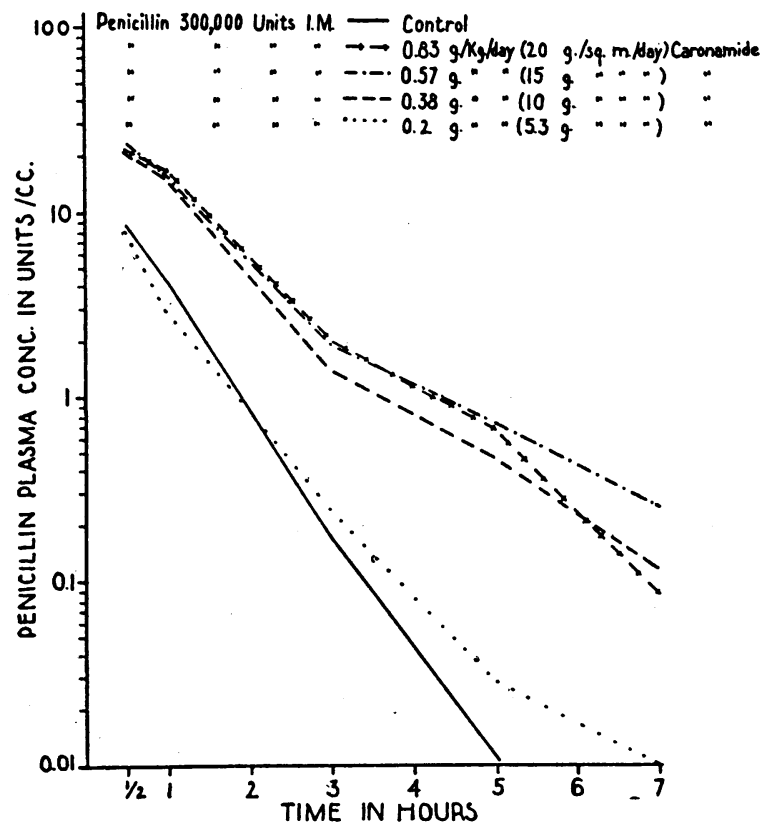

Fig. 1. Patient A. A. Characteristic Penicillin Excretion Curves with Different Doses of CarONAMIDE

7 The question of disappearance of penicillin by means of inactivation by the binding properties of plasma proteins does not materially modify this assumption in the case of penicillin G. Tompsett, Shultz and McDermott (14) have shown that 84 per cent of injected penicillin $G$ is excreted in the urine.

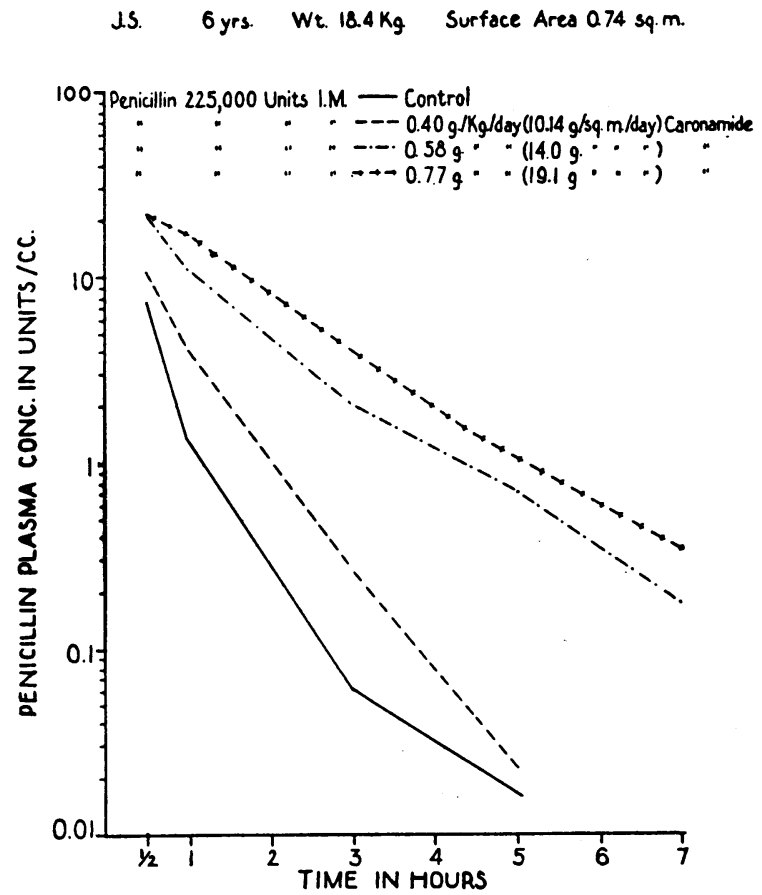

Fig. 2. Patient J. S. Characteristic Penicillin Excretion Curves - with Different Doses of CarONAMIDE

maximum decrease of excretion rate was taken as the dose causing complete suppression of tubular excretion (see Figures 1 and 2). For the purpose of convenience, these clearance rates are given numerical values as calculated from a standard formula, ${ }^{8}$ and are referred to as constants of excretion rates $(K)$. (See Tables I and II.)

8 The formulae used for calculating (1) the slope of the decrease of the measured plasma penicillin levels and (2) the slope of the calculated decrease of penicillin levels expected with glomerular filtration alone, are given below.

In both, $K=$ the constant rate of clearance of the extracellular fluid of penicillin by the kidney in a given unit of time.

Formula 1:

The standard formula (15) for the calculation of the rate of decrease of the plasma concentration of a substance by means of a unimolecular reaction is $-\frac{d c}{d t}=K c$. Here $c=$ the concentration of penicillin, $K=$ proportionately factor or constant, and $-\frac{d c}{d t}=$ rate at which the penicillin concentration decreases. By integration this becomes:

or

$$
\begin{aligned}
& K=\frac{\log _{e} C_{1}-\log _{e} C_{2}}{t_{2}-t_{1}} \\
& K\left(t_{2}-t_{1}\right)=\log _{e} \frac{C_{1}}{C_{2}}
\end{aligned}
$$


TABLE I

Patient A. A.

Grams of Caronamide/
$\mathbf{K g} . / 24$ hours
0. (control)
0.2
0.38
0.57
0.83
Estimate complete
suppression

$\begin{gathered}\text { Constants of excretion } \\ \text { rates }(K)\end{gathered}$
1.61
1.18
0.94
0.82
0.85
0.82

A second indirect approach was also used. It has been shown by Newman et al. (8) that there is a direct mathematical relationship between the rate of decrease of the plasma concentration of a substance, the rate of glomerular filtration, and the volume of the extracellular fluid compartment. Using this relationship it was possible to calculate the rate of decrease of plasma penicillin concentration that might be expected if only glomerular filtration were occurring. The calculation was made according to a standard formula ${ }^{8}$ in which the normal average glomerular filtration rate was

$\begin{array}{cc}\text { Let } & t=\text { time in hours, } \\ \text { and let } & t_{2}-t_{1}=1 . \\ \text { Then } & K=\log _{e} \frac{C_{1}}{C_{2}} .\end{array}$

Converting to common log for direct comparison with the plotted graphs

$K=\log \frac{C_{1}}{C_{2}} \times 2.3$

Formula 2:

(2.303 = factor for converting natural logarithm to common logarithm)

The slope of the curve $(K)$ which would exist if penicillin were excreted by glomerular filtration alone can be calculated from the following formula derived from the mathematical relationship existing between plasma concentration, extracellular fluid volume and the rate of glomerular filtration in cc./min. (8).

$K=\frac{R}{V}$ where $R$ is the glomerular filtration rate and $V$ is the volume of extracellular fluid.

Since the plasma concentrations measured in Formula 1 were taken at hourly intervals, $R$ was taken to equal 120 (average cc./min.) $\times 60$.

$V=20$ per cent of body weight in kilograms, expressed as cubic centimeters.

Since the average glomerular filtration rate was derived from adults with an average surface area of 1.7 meters, the filtration rate of each child was converted to the same basis by multiplying $R$ by the area in square meters of the child $(A)$ divided by 1.7 .

The final formula then was:

$$
K=\frac{120 \times 60}{20 \text { per cent body wt. in Kg. as cc. }} \times \frac{A}{1.7} .
$$

TABLE II

Patient J.S.

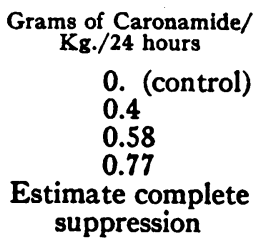

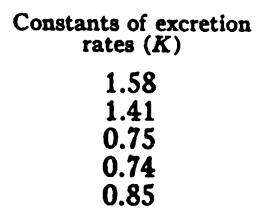

used $(120 \mathrm{cc} . / \mathrm{min}$.) and the extracellular fluid volume was assumed to be 20 per cent of the body weight. In this way, a theoretical constant $(K)$ for the rate of plasma penicillin glomerular clearance was obtained for each child. This was used as a standard with which the constant of the slowest rate obtained with Caronamide in the same child was compared. Complete identity of the measured slope with, or a rate slower than, the estimated standard slope was taken as 100 per cent tubular suppression; faster rates were expressed as percentage of tubular suppression attained by that dose of Caronamide.

The graphic representation of the excretion slopes with Caronamide also demonstrates the delayed excretion rate in terms of the time of disappearance of penicillin from the blood stream. In two of three cases, levels of over 0.02 units/cc. were still present at 12 hours.

As previously mentioned, the Caronamide dosage was based on the body surface area as a unit of measurement because this is known to be more closely correlated with renal function than is body weight. Since, however, this is an uncommon unit in clinical practice, it was thought advisable to recalculate the doses in terms of body weight.

Figure 3 is a graphic summary of the findings. At a dose of $0.2 \mathrm{Gm} . / \mathrm{Kg} . / 24$ hours, there is no appreciable suppression except in one case. At approximately $0.4 \mathrm{Gm} . / \mathrm{Kg} . / 24$ hours, there is a wide scattering of effect from 23 to 100 per cent, with an average suppression of 56.4 per cent. Between 0.55 and $0.70 \mathrm{Gm} . / \mathrm{Kg} . / 24$ hours, there is suppression of from 71 to 100 per cent, with an average of 91 per cent. Fifty per cent of these trials are estimated as showing total suppression. Between 0.70 and $0.92 \mathrm{Gm} . / \mathrm{Kg} . / 24$ hours, the average suppression is 97 per cent. From these data, 0.55 to $0.70 \mathrm{Gm}$. of Caronamide $/ \mathrm{Kg} . / 24$ hours appears to represent an effective dosage range of this drug in the age-group studied. On 


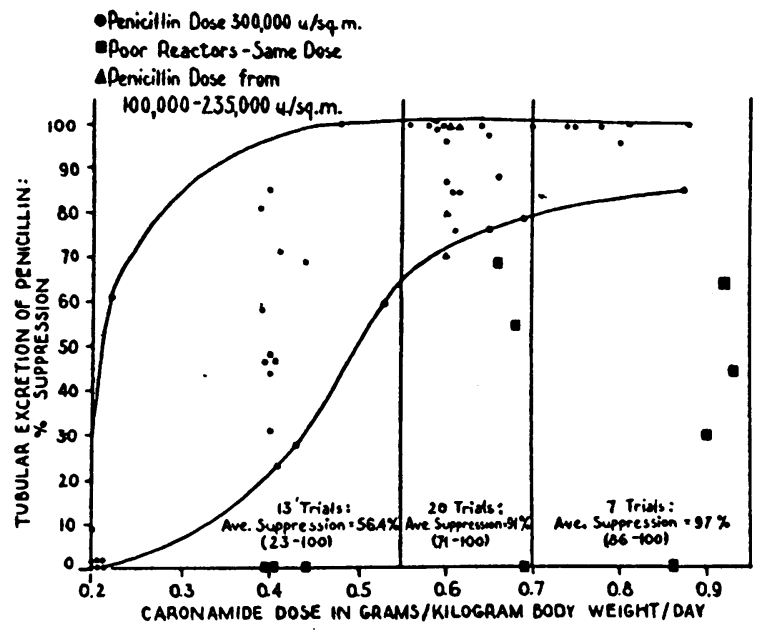

Fig. 3. Tubular Excretion of a Fixed Dose of Penicillin with Increasing Doses of Caronamide PER KG. OF BOdY WEIGHT

The lines represent the upper and lower limits of the percentage suppression obtained with a. Penicillin dosage of $300,000 \mathrm{U} / \mathrm{sq}$. M.

the basis of surface area, the younger children in the age-groups studied will probably require the larger doses per $\mathrm{Kg}$. within this range, whereas

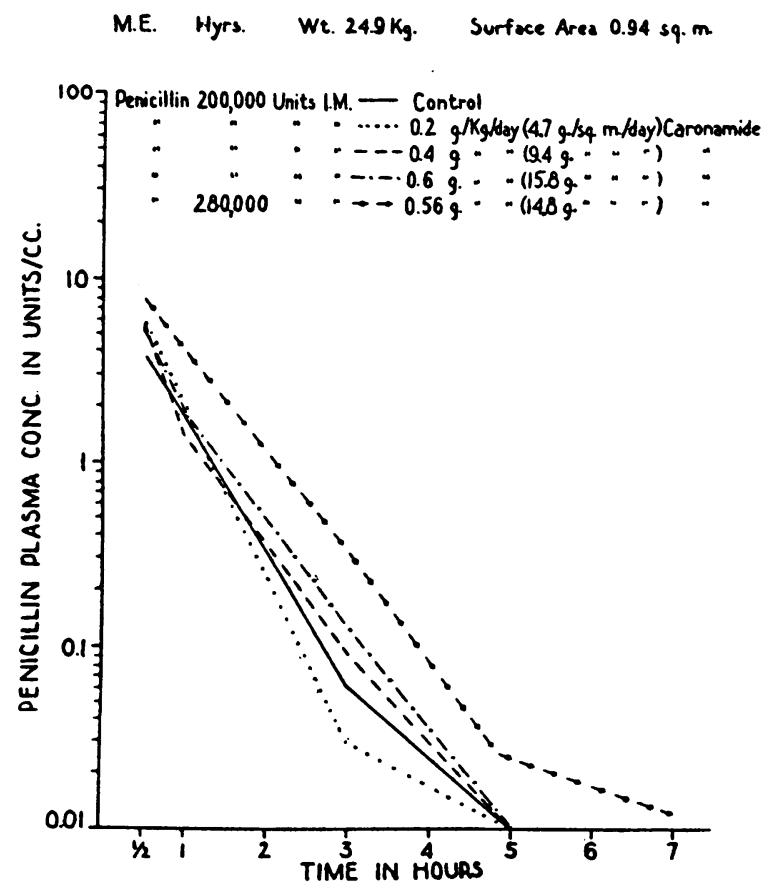

Fig. 4. Patient M. E. Penichlin Excretion Curves Consistently Fail to Show More than a Minimal Decrease in Rate even with Caronamide Doses within the Normally Effective Rayge older children, above the weight of $27 \mathrm{Kg}$., will probably need progressively smaller doses per $\mathrm{Kg}$. within the range. In still older children, it would be reasonable to use the adult dosage schedule recommended by Boger (9), of a total of $24 \mathrm{Gm} . / 24$ hours, in six or eight divided doses.

Effective tubular suppression was not obtained in some children, even with Caronamide doses higher than those in this range. One of these is illustrated in Figure 4. A few individuals, such as the one shown in Figure 5, responded incon-

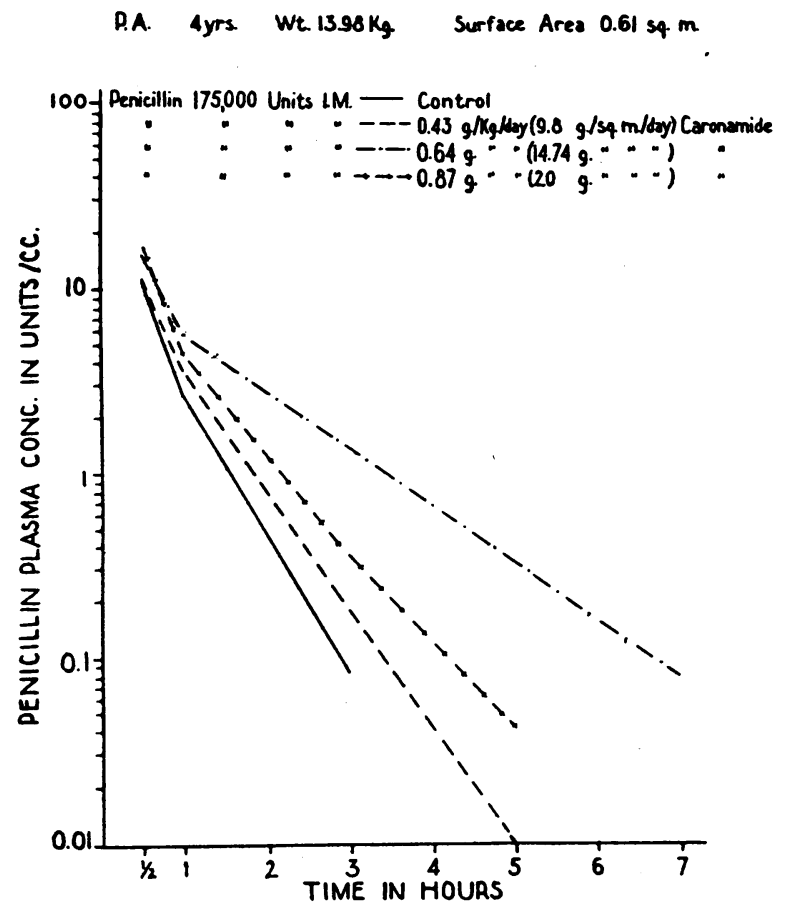

Fig. 5. Patient P. A. Penichlin Excretion Curves Show an Inconsistent Response to Doses of Caronamide in the Normally Effective Range

The response to the largest dose is less than to a smaller dose.

sistently ; in this instance a dose of $0.8 \mathrm{Gm} . / \mathrm{Kg} . /$ 24 hours produced less effect than a lower dose of $0.6 \mathrm{Gm} . / \mathrm{Kg} . / 24$ hours, which caused complete suppression. In patient M. E., illustrated by Figure 4, only a minimal improvement over the control was obtained in four trials at varying doses up to $15 \mathrm{Gm}$./sq. M. These failures are apparently due to variability of Caronamide $a b-$ sorption. This was substantiated by finding in patient M. E. a urinary excretion of only 0.25 per cent of the ingested Caronamide over a 28-hour pe- 
riod, whereas a consistently good reactor (patient A. A., Figure 1) showed a 24-hour excretion of 60 per cent and 67 per cent of ingested Caronamide, respectively, in two trials. In the poor reactor (patient M. E.) no Caronamide was demonstrated in the plasma in two out of three trials; a plasma concentration of less than $5 \mathrm{mg}$. per cent on the third trial was present. In adults, Boger (9) has shown that a plasma Caronamide concentration of 20 to $40 \mathrm{mg}$. per cent was required to give satisfactory blocking of the tubular excretion of penicillin. Of a total of 23 trials ( 15 patients) at doses within the recommended range, 0.55 to $0.70^{\circ} \mathrm{Gm} . / \mathrm{Kg} . / 24$ hours, significant tubular suppression was obtained in 20 trials (12 patients), with no response or inadequate response in three (three patients). Thus, a satisfactory response was obtained in 87 per cent of the trials.

Figure 6 illustrates that with an effective dose of Caronamide the rate of penicillin excretion, at three different dosage levels, is the same. The levels reached by 100,000 units with Caronamide are rather better than those reached by three times the dose without Caronamide. Figure 7 shows that with an effective dose of Caronamide,

A. 9yrs Wt. $2636 \mathrm{~kg}$ Surface Area $1.02 \mathrm{sq} \cdot \mathrm{m}$.

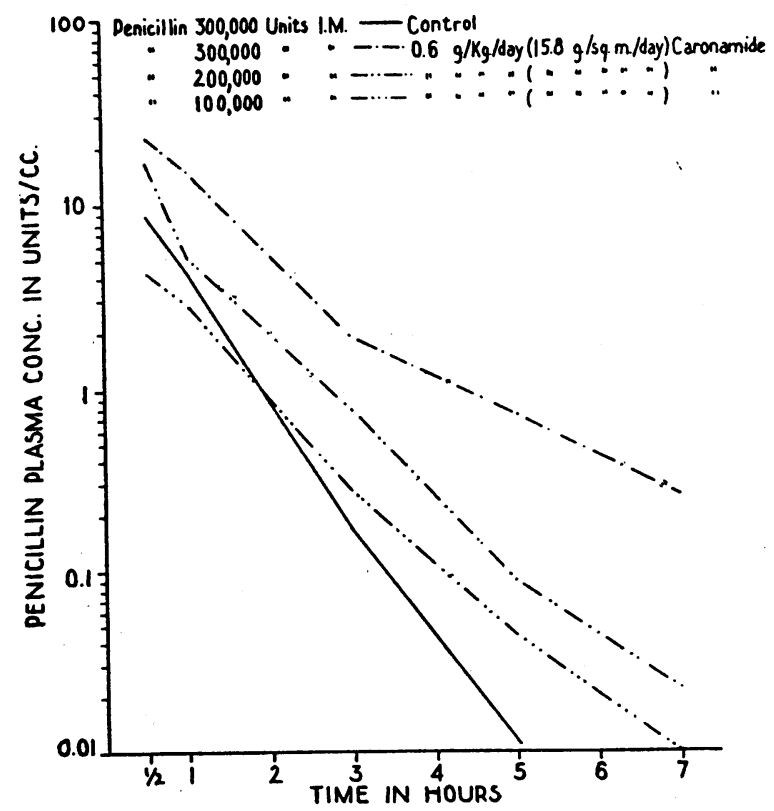

Fig. 6. Patient A. A. Comparison of Penicillin Excretion Curves of Three Different Penicillin Doses Using an Optimal Dose of Caronamide

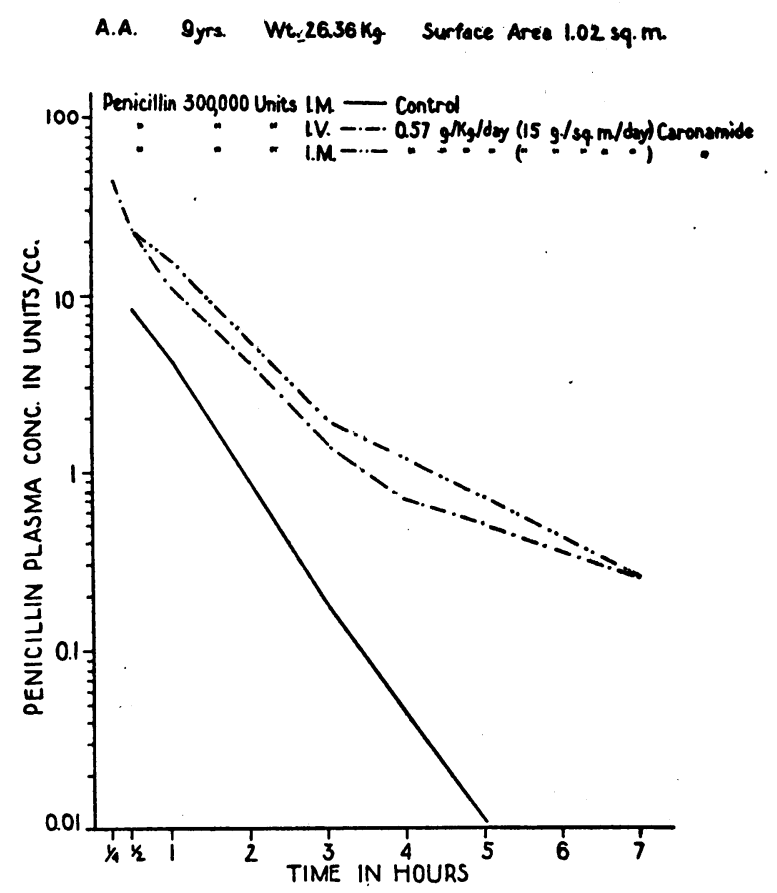

Fig. 7. Patient A. A. Comparison of the Curves of Penicillin Excretion after Intravenous and INtramuscular Administration Using an Optimal Dose of Caronamide

the excretion of penicillin given intravenously is at the same rate as that given intramuscularly. An identical result was obtained in three different patients.

\section{Toxicity}

In this study, toxicity of Caronamide was found to be of a very low order, confirming the impression obtained in earlier work (5). Toxic reactions were encountered on only five occasions out of the 59 tests conducted on 21 patients. In four patients, these consisted of anorexia, nausea and vomiting, and then only at doses higher than the dose range now considered advisable. In three of these children, there was no reaction with high daily doses given at four-hour intervals, but the same daily dose given at six-hour intervals with correspondingly larger individual doses produced nausea and vomiting. In the fifth patient a transient macular itching rash appeared over the face, neck, and shoulder girdle after nine doses of Caronamide. This began to fade four hours after discontinuing the drug and had disappeared within 36 hours. 
It seems important to record here two severe sensitivity reactions observed after this series was completed: One was a $2 \frac{1}{2}$-year-old white boy, R. J. (chart No. 46579), who had a seborrhoic dermatitis associated with marked sensitivity to the staphylococcus aureus. $\mathrm{He}$ was given one course of penicillin and Caronamide lasting 16 days without apparent reaction. Six days later, he was started on a second course of Caronamide at a dosage of as low as $0.27 \mathrm{Gm} . / \mathrm{Kg} . / 24$ hours. After two doses of $1.25 \mathrm{Gm}$. each, he developed an extensive maculo-papular rash with a rectal temperature of $103^{\circ} \mathrm{F}$. A white count done at the height of this reaction showed a slight polymorphonuclear leucocytosis. The reaction faded within 24 hours of stopping medication. A test dose given five days later led to a similar but more severe reaction. The second was a white boy of three years, D. T. (chart No. 46387), being treated for subacute bacterial endocarditis. He was given Caronamide $(0.6 \mathrm{Gm} . / \mathrm{Kg} . / 24$ hours $)$ for two days without reaction. Six days later, he was given one dose of $1.5 \mathrm{Gm}$. Four hours later, he developed rigors, a temperature of $105^{\circ} \mathrm{F}$. and a diffuse erythema. All these signs subsided in 12 hours without therapy.

\section{DISCUSSION}

The data presented suggest that in children of the age range studied, two to nine years, complete or almost complete suppression of the tubular excretion of penicillin can be achieved in over 85 per cent by the oral administration of optimal doses of Caronamide. The result of such a suppression is to raise the plasma concentration of penicillin at any given dose and to prolong the time during which such a level is maintained. It would appear that in clinical medicine this fact would be valuable in a situation where the patient is infected with a comparatively resistant organism, since very high blood levels can be attained and maintained by suitably adjusting the size and frequency of penicillin administration in conjunction with the recommended dosage of Caronamide. This treatment has been used effectively for resistant cases of subacute bacterial endocarditis $(10,11)$. It also may be of value in reducing the need for frequent doses of penicillin by prolonging the maintenance of a theoretically effective blood level for organ- isms of all degrees of sensitivity. A single dose of penicillin per 24 hours may be sufficient for very sensitive organisms. The results of Zubrod's work (12) suggest, however, that infrequent but large doses of penicillin may be effective therapeutically without the addition of Caronamide. It is not within the scope of this paper to discuss the best clinical application of these data since this must be determined by actual clinical trial. It may well be that smaller doses of Caronamide, at longer intervals, giving less than complete tubular suppression will be entirely adequate for the treatment of most infections. On the other hand, it seems more probable that the need for Caronamide may be confined to those situations just mentioned, in which very high and prolonged blood levels are needed for the therapy of infections by resistant organisms. With the recent development of a method for determining blood levels of Caronamide, a closer correlation between therapeutic effect and blood levels will be possible. Using this method, Boger (9) has found that there is a tendency for Caronamide to accumulate in the blood so that the dosage schedules may have to be adjusted according to blood levels. Also some method may be found of improving the absorption of Caronamide from the intestinal tract and thus make the drug valuable to the 15 per cent of patients who now fail, temporarily or permanently, to respond. In this study, no attempt has been made to evaluate the dosage for infants under two years of age. The recognized inefficiency of the immature kidneys of infants up to six months at least (13) would suggest that smaller doses of Caronamide would be needed in this age-group to effect the same results on the penicillin excretion rate.

\section{SUMMARY}

A comparison of the excretion rates of penicillin given intramuscularly alone and with varying oral doses of Caronamide was used to determine the dose of Caronamide effecting complete suppression of tubular excretion.

By this method, an estimated 71 to 100 per cent, with an average of 91 per cent, suppression was achieved in 20 trials with doses of Caronamide between 0.55 and $0.70 \mathrm{Gm} . / \mathrm{Kg} . / 24$ hours in children between two and nine years. 
Of a total of 23 trials at doses within this range, such a suppression of tubular function failed to occur in three. This was apparently due to a failure of absorption of Caronamide from the gastrointestinal tract.

Suppression of tubular excretion led to the maintenance of effective blood levels of penicillin for as long as six to eight hours after the administration of a dose of approximately 10,000 units/ $\mathrm{Kg}$.

Toxic symptoms of anorexia, nausea, and vomiting were encountered in four patients only with doses higher than those finally recommended. One mild drug rash was encountered in this series. Two other children, not in the series, were observed to have a severe sensitivity reaction resembling that encountered with the sulfonamides.

\section{BIBLIOGRAPHY}

1. Beyer, K. H., New concept of competitive inhibition of the renal tubular excretion of penicillin. Science, 1947, 105, 94.

2. Beyer, K. H., Miller, A. K., Russo, H. F., Patch, E. A., and Verwey, W. F., The inhibitory effect of Caronamide on the renal elimination of penicillin. Am. J. Physiol., 1947, 149, 355.

3. Beyer, K. H., Russo, H. F., Patch, E. A., Tillson, E. A., and Shaner, G., Certain pharmacologic properties of 4-carboxyphenyl methanesulfonanilide including its effect on renal clearance of compounds other than penicillin. J. Pharm. \& Exper. Ther., 1947, 91, 272.

4. Verwey, W. F., and Miller, A. K., The effect of Caronamide upon penicillin therapy of experimental pneumococcus and typhoid infections in mice. Proc. Soc. Exper. Biol. \& Med., 1947, 65, 222.

5. Rapoport, M., Corneal, F. B., Beyer, K. H., and Verwey, W. F., A clinical evaluation in children of the toxicity and efficacy of Caronamide for the competitive inhibition of penicillin excretion. Am. J. M. Sc., 1948, 215, 514.

6. Rammelkamp, C. H., A method for determining the concentration of penicillin in body fluids and exudates. Proc. Soc. Exper. Biol. \& Med., 1942, 51, 95.

7. Eagle, H., and Newman, E. V., The renal clearance of penicillins $F, G, K, \& X$, in rabbits and man. J. Clin. Invest., 1947, 26, 903.

8. Newman, E. V., Bordley, J., III, and Winternitz, J., The interrelationships of glomerular filtration rate (Mannitol clearance) extracellular fluid volume, surface area of the body, and plasma concentrations of Mannitol. Bull. Johns Hopkins Hosp., 1944, 75, 253.

9. Boger, W. P., Caronamide, a new enhancing agent for use in conjunction with penicillin therapy. Tr. \& Stud. of the Coll. Physicians of Phila., 1947, 15, 104.

10. Loewe, L., Eiber, H. B., and Alture-Werber, E., Enhancement of penicillin blood levels following oral administration of Caŗonamide. Science, 1947, 106, 494.

11. Boger, W. P., Kay, C. F., Eisman, S. H., and Yeoman, E. E., Caronamide, a compound that inhibits penicillin excretion by the renal tubules, applied to the treatment of subacute bacterial endocarditis. Am. J. M. Sc., 1947, 214, 493.

12. Zubrod, C. G., Comparative efficiency of single and multiple dosage regimens of the penicillins. Bull. Johns Hopkins Hosp., 1947, 81, 400.

13. West, J. R., Smith, H. W., and Chasis, H., Glomerular filtration rate, effective renal blood flow, and maximal tubular excretory capacity in infancy. J. Pediat., 1948, 32, 10.

14. Tompsett, R., Shultz, S., and McDermott, W., Influence of protein-binding on the interpretation of penicillin activity in vivo. Proc. Soc. Exper. Biol. \& Med., 1947, 65, 163.

15. Getman, F. H., and Daniels, F., Outlines of Theoretical Chemistry. John Wiley \& Sons, London. 1931, Ed. 5, p. 322. 